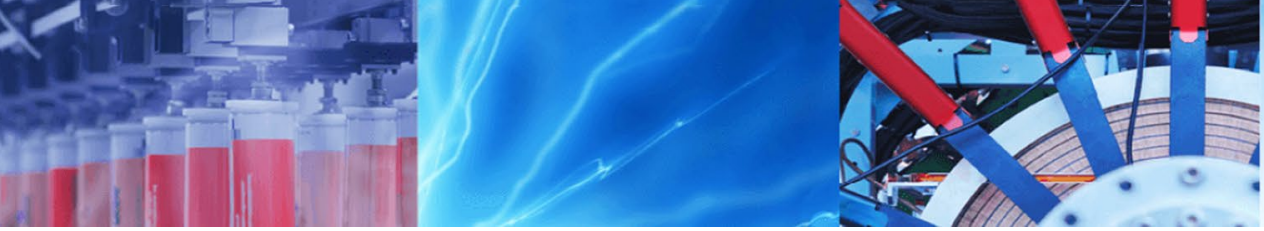

Research Article

\title{
Compatibility of poly(dimethylsiloxane) microfluidic systems with high viscosity hydrocarbons
}

\author{
Kiarash Keshmiri $^{1} \cdot$ Haibo Huang ${ }^{2} \cdot$ Neda Nazemifard ${ }^{1}$ (D)
}

(c) Springer Nature Switzerland AG 2019

\begin{abstract}
In this work, the compatibility of poly(dimethylsiloxane) (PDMS) with high viscosity petroleum liquids (bitumen) was evaluated to study the possibility of using PDMS microchannels in heavy oil and bitumen extraction research using solvent based methods such as Vapor Extraction. Three curing agent to base ratios (1:2, 1:10, and 1:20) were used to fabricate PDMS. Swelling ratio of these three samples in the vicinity of different organic solvents and diluted bitumen were evaluated by measuring the weight of sample every $15 \mathrm{~min}$. It was found that 1:10 ratio PDMS was preferential ratio for minimizing absorption. We were particularly interested in the kinetic of swelling, deformation, and discoloration of PDMS. The hypothesis was that a PDMS microchip can still be used for experiments with heavy oil and bitumen if the experiment time is much shorter than the time it takes for PDMS discoloration, swelling, and deformation. The coating of PDMS slabs with trichloro ( $1 \mathrm{~h} 1 \mathrm{~h} 2 \mathrm{~h} 2 \mathrm{~h}$-perfluorooctyl) silane was also tested and swelling ratios were again measured to evaluate the effect of surface coating. Swelling ratios were at the same order for uncoated PDMS which shows silane coating is not able to improve the solvent compatibility of PDMS. Moreover, solubility parameter was used to predict the swelling ratio of 1:10 PDMS sample in organic solvents. It predicts that hexane and toluene have the highest solubility that was in great agreement with experimental results. It seems that solubility parameter is a reliable factor to qualitatively predict the swelling ratio of PDMS. The effect of bitumen on transparency of PDMS was also studied using Ultraviolet-Visible spectrophotometer.
\end{abstract}

Keywords Microfluidics · Poly(dimethylsiloxane) $\cdot$ Polymers · Swelling · Enhanced oil recovery $\cdot$ Chemical compatibility

\section{Introduction}

Microfluidic devices have been applied in petroleum or energy [1-4] for evaluation of wetting properties [5], single [6] and multiphase flow study [7], asphaltene deposition $[8]$, enhanced oil recovery $[9,10]$ or other fields like fuel cells [11], microbioreactors [12], etc. Glass was the main substrate for micro fabrication early in 1990 s due to its mechanical and optical properties, low chemical reactivity, and transparency and they are mostly fabricated by photolithography and wet etching methods [13]. However, there are number of disadvantages for glass microchannels like high price and multistage fabrication process and irreproducible thermal bonding process [14]. Poly(dimethylsiloxane) (PDMS) is one of the most popular materials for microfluidic fabrication that has been thoroughly studied in various fields of application [15-17]. PDMS microchannels are low-cost and easy to fabricate with low polarity and chemical inertness [18-20]. Moreover, they are transparent to visible light and suitable for detection systems like ultraviolet-visible (UV-Vis) and fluorescence [21]. PDMS consists of base and curing agent that various curing agent to base ratios alter the properties of elastomer such as stiffness [22-24].

\footnotetext{
$\triangle$ Neda Nazemifard, nn1@ualberta.ca; Kiarash Keshmiri, kiarash@ualberta.ca; Haibo Huang, haibo.huang@innotechalberta.ca| ${ }^{1}$ Department of Chemical and Materials Engineering, University of Alberta, Edmonton, AB T6G 2V4, Canada. ${ }^{2}$ Reservoir and Geosciences, InnoTech Alberta, 250 Karl Clark Road NW, Edmonton, AB T6N 1E4, Canada.
} 
One of the main drawbacks of PDMS is that it swells with solvents such as toluene and low molecular weight organic solutes. In addition, bitumen as a complicated material with various fractions and large molecules such as asphaltene can affect the swelling ratio. Although accurate knowledge of the dimensions is very important in analysis of data captured from microchannel and fluid flow, swelling deforms the microchannel and change the rate and profile of the flow, consequently. Therefore, compatibility evaluation of PDMS with organic solvents along with heavy oil and bitumen [25] are the basic requirements in specific energy related fields such as capillary driven flow and pore scale study of effective forces on bitumen extraction where solvents are used for viscosity reduction. Noteworthy to mention that our hypothesis for this work was that if the swelling rate is slow, we can still use PDMS as a cheap and easily-made choice for microfluidic studies where the experimental time (i.e. contacting time of PDMS and bitumen) is shorter than swelling rate of the chip.

There are a number of surface modification methods to improve the solvent compatibility of the PDMS. Abate et al. [26] presented a coating technique with a glass-like layer using sol-gel chemistry. It increases chemical resistance of the channel, but it changed the dimensions of the designed microchannel. Coating of the surface with other materials like Teflon [27] and silane [28] are reported by other authors. Laser surface modification [29] is another method to adjust the surface but considering this technique, PDMS is not a cheap and fast method of microfluidic device fabrication anymore. Application of self-assembled monolayer (SAM) with a high number of $\mathrm{CF}_{3}$ and $\mathrm{CF}_{2}$ groups alter the wettability of the surface [30]. Presence of these functional groups on the surface of FOTS coated PDMS has been reported by other authors [30]. There are a few limitations on PDMS surface treatment such as aggregation of fluorocarbon and surface structural defects that impacts the performance of the coating [31].

In this study, dynamic of swelling ratio of PDMS in pure solvents and diluted bitumen samples (10-50\%) in small time-lapsed increments were measured and transparency of swelled PDMS as a function of time was evaluated using UV-Vis. Total Internal Reflection Fluorescence (TIRF) microscopy was also applied to detect the bitumen on PDMS samples due to the fluorescence nature of the bitumen [32]. Moreover, PDMS slabs were coated with trichloro( $1 \mathrm{~h} 1 \mathrm{~h}$ $2 \mathrm{~h} 2 \mathrm{~h}$-perfluorooctyl) silane (FOTS) in order to evaluate the effect of silane coating on solvent and bitumen compatibility of PDMS.

\section{Theory}

Solubility parameter can correlate experimental swelling based on cohesive energy densities of the materials. For two materials to be soluble, their cohesive energy densities must be similar, since this energy must be overcome to separate the molecules of the solute to allow the molecules of solvent to insert. Therefore, solubility parameter is useful for predicting the swelling behavior of a polymer in a solvent without knowing any other information about the solvent [18]. For a binary system the Hildebrand-Scatchard equation [33] relates the solubility parameters of non-polar liquids to the enthalpy change on mixing them:

$\Delta H_{m}=V_{m}\left(\delta_{1}-\delta_{2}\right)^{2} \emptyset_{1} \emptyset_{2}$

where $V_{m}$ is the volume of the mixture, $\delta_{i}$ is the solubility parameter of the component $i$, and $\emptyset_{i}$ is the volume fraction of $i$ in the mixture. For a soluble binary system, the free energy of mixing must be less than zero [34]. Based on the energy, enthalpy, and entropy of mixing $\left(\Delta G_{m}=\Delta H_{m}-T \Delta S_{m}\right)$, and Hildebrand-Scatchard equation $\left(\Delta H_{m} \infty\left(\delta_{p d}-\delta_{s}\right)^{2}\right)$, swelling is maximal when $\left(\delta_{p}-\delta_{s}\right)^{2}$ is zero or solubility parameter of PDMS $\left(\delta_{p d}\right)$ and solubility parameter of solvent $\left(\delta_{s}\right)$ are similar [33]. However, there are some exceptions for maximal swelling because of other effective factors on such as polarity of the solvent [35]. Hansen [36] divided solubility parameter into dispersion forces $\left(\delta_{d}\right)$, polar forces $\left(\delta_{p}\right)$, and hydrogen bonding forces $\left(\delta_{h}\right)$ within the material (Eq. 2). The role of each parameter can affect the strength of the solubility even at the same solubility parameters for two solvents.

$\delta^{2}=\delta_{d}^{2}+\delta_{p}^{2}+\delta_{h}^{2}$

Su et al. [37] showed that polar interaction is the main effect for deciding the swelling degree of PDMS in water and alcohol. This is in good agreement with our results because hexane and toluene had the highest swelling ratio and polar section of the solubility parameter is the main factor that is close to PDMS polar parameter. Lee et al. [18] reported an indistinguishable solubility parameter of $9.9 \mathrm{cal}^{1 / 2} \mathrm{~cm}^{-3 / 2}$ for methylene chloride and acetone with much higher swelling of methylene chloride compared with acetone that is related to the polarity of the solvents. The solubility of solvents in PDMS is also determined with interaction radius $\left(R_{i}\right)$ (Eq. 3) that has the same unit as solubility parameter [38]. The smaller the $R_{i}$, the higher the solubility of the solvent into PDMS [39].

$$
\begin{aligned}
R_{i}^{2}= & 4\left(\delta_{\text {solute }, d}-\delta_{\text {solvent }, d}\right)^{2}+\left(\delta_{\text {solute }, p}-\delta_{\text {solvent }, p}\right)^{2} \\
& +\left(\delta_{\text {solute }, h}-\delta_{\text {solvent }, h}\right)^{2}
\end{aligned}
$$


It is noteworthy to mention that experimental swelling ratio of PDMS at room temperature (i.e. $22^{\circ} \mathrm{C}$ ) was measured using the following equation where $w$ is the mass of swollen network and $W_{0}$ is the mass of dry extracted PDMS slab.

Swell ratio $=\frac{w-w_{0}}{w_{0}}$

\section{Materials and method}

\subsection{Material}

Hexane (lab grade), acetone (lab grade), methanol (analytical grade), toluene (HPLC grade), and isopropanol (lab grade) were supplied from Sigma-Aldrich Co., USA. PDMS (Sylgard 184 Silicone Elastomer Kit) purchased from Dow Corning Co., USA. Trichloro(1 h 1 h 2 h 2 h-perfluorooctyl) silane (FOTS) was supplied from Sigma-Aldrich Co., USA. McKay River bitumen was provided by Innovates Alberta.

\subsection{PDMS fabrication and bitumen sample preparation}

According to the manufacturer (Dow Corning, Midland, $\mathrm{MI}$ ), the recommended ratio for curing agent to base is $1: 10$. Here we have tried two other ratios with higher curing agent $(1: 2)$ and less $(1: 20)$ to evaluate the optimum ratio for PDMS fabrication. Therefore, PDMS samples were prepared by mixing a 1:2, 1:10, and 1:20 curing agent to base (Sylgard 184) ratios. Higher curing agent:base ratio means higher cross linking and rigid PDMS structure that breaks easily under shear strain. Mixture was degassed in a vacuum chamber for $60 \mathrm{~min}$ to remove the air trapped during the mixing. Then mixture was poured into a brass mold with two cylindrical reservoirs and a $20 \mathrm{~mm} \times 2 \mathrm{~mm} \times 100 \mu \mathrm{m}$ (length $\times$ width $\times$ depth) rectangular channel connecting the reservoirs and cured for $24 \mathrm{~h}$ at $80^{\circ} \mathrm{C}$ to add $\mathrm{Si}-\mathrm{H}$ bonds to $\mathrm{C}=\mathrm{C}$ bonds. PDMS was extracted and individual chips was washed and glass substrate $(24 \times 50$ microscope coverslips from Fisher Scientific) was bonded to the PDMS forming an enclosed space. Glass substrates were cleaned using piranha solution $\mathrm{H}_{2} \mathrm{SO}_{4}$ and $\mathrm{H}_{2} \mathrm{O}_{2}$ with 3:1 volume ratio) and rinsed using de-ionized water. Cleaned PDMS and glass substrate wafers were pressed together and baked in oven at $80^{\circ} \mathrm{C}$ for $1 \mathrm{~h}$ for the reversible sealing using van der Waals forces at room temperature. A drop of fluid sample $(\sim 15 \mu \mathrm{l})$ was placed in the inlet reservoir and fluid flowed through the micromodel due to capillary forces. In addition to microchannels, PDMS samples were cut into small square pieces $(2.5 \mathrm{~cm} \times 2.5 \mathrm{~cm})$ to submerge in different liquids. Pieces were washed with acetone and then DI water to remove the possible contaminants. Stock bitumen was used to prepare $10-50 \%$ bitumen dilutions in hexane. All the samples were aged for $24 \mathrm{~h}$ to settle down the large aggregates and precipitates.

\subsection{Silanization}

Effect of silanization on swelling ratio of PDMS slabs in solvents were evaluated by coating the PDMS surface with FOTS. In fact, this coating is a fast and cheap process for surface modification that can improve the possibility of using PDMS microchannels in heavy oil extraction using solvent. For silanization, a small amount of FOTS was placed in a vacuum chamber for $4 \mathrm{~h}$ at room temperature (i.e. $22^{\circ} \mathrm{C}$ ). Evaporated silane molecules react with surface silanols to form siloxane bonds at the interface. Irreversible bonding forms a monolayer of silane that was confirmed by water contact angle measurement that increased from $\sim 95^{\circ}$ (untreated PDMS) to $\sim 115^{\circ}$ (silanized PDMS) as shown in Fig. 1. Noteworthy to mention that PDMS slabs were washed with piranha solution for activation to generate surface silanol groups before silanization process [30].

\subsection{UV-Vis spectrophotometer}

The transparency of the PDMS swollen at different bitumen samples was measured using UV-Vis spectrophotometer (Cary 50 UV-Vis, Varian Inc., USA) at a wavelength of $400 \mathrm{~nm}$ and room temperature $\left(22^{\circ} \mathrm{C}\right)$. For this purpose, transparency of $2.5 \mathrm{~cm} \times 1 \mathrm{~cm} \times 0.3 \mathrm{~cm}$ (length $\times$ width $\times$ thickness) PDMS slabs were measured as a function of time. Absorbance (A) was calculated and related to the transmittance (T) based on the Eq. (2).

$A=\log _{10}\left(\frac{100}{T(\%)}\right)=2-\log (T \%)$

\subsection{TIRF microscopy}

In this work, TIRF microscopy was applied to image absorbed bitumen on the surface layer of the PDMS due to its the natural fluorescence [42]. This method excites fluorescence at solid surface without any other fluorescence background from deeper areas [32]. Therefore, fluorescence nature of the bitumen enables us to capture absorption of bitumen on the surface of the sample. As mentioned earlier, TIRF microscopy eliminates background noises of the PDMS. In fact, PDMS has small pores on the surface and TIRF microscope illustrates bitumen distribution on specific spatial location. Based on previous study in our group [32], a $488 \mathrm{~nm}$ laser source and 500-550 nm (green) emission filter were selected for the microscope. 

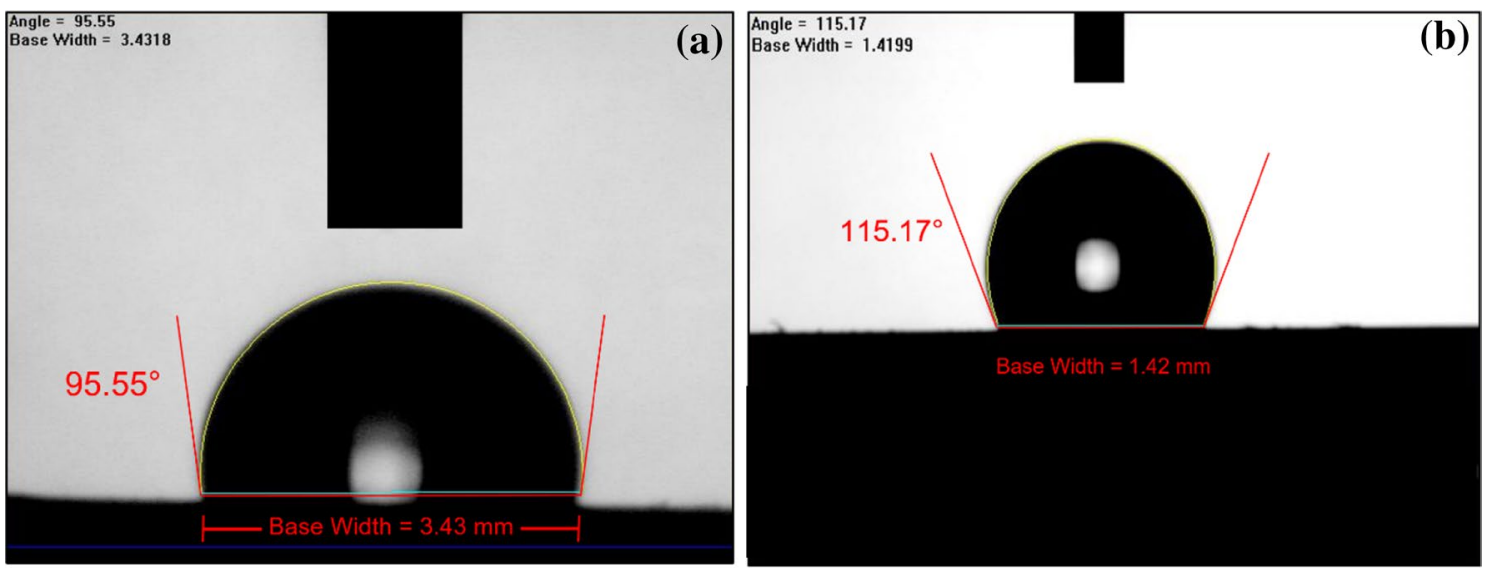

Fig. 1 Water contact angle on a pure PDMS and $\mathbf{b}$ FOTS treated PDMS slab

\section{Result and discussion}

\subsection{Pure solvents swelling}

The first part was related to the effects of PDMS preparation method on solubility in different solvents. Therefore, weight of swollen networks at different reagent to base ratios (1:10, 1:2, 1:20) were recorded. All sides of PDMS slabs should be covered with solvent for a uniform absorption of the solvent. Swelling ratio of the PDMS in solvent should be measured at equilibrium condition. This condition was considered at the time that there are no more changes in the mass of swollen network. Based upon this, a procedure was developed whereby small $(5 \mathrm{~cm} \times 5 \mathrm{~cm})$ squares of PDMS were immersed in solvent in airtight bottles. Due to the volatile nature of the exposed to air solvents at room temperature $\left(22^{\circ} \mathrm{C}\right)$, the evaporation of the solvent could affect the measurements. This was resolved by measuring the weight of samples while it was submersed in the solvent plate. Weight of samples were measure in different time intervals until a constant weight was observed.

Swelling ratio of PDMS samples $(1: 2,1: 10$, and 1:20 ratios) in different solvents was depicted in Fig. 2. Slabs were submerged into solvent and weight of samples were measured at different times until there were no more changes in PDMS weight. Each experiment was performed for three times to evaluate the reliability of the results and error bars were calculated based on repeatability of the experiments with a $95 \%$ confidence interval of the mean. According to the error bars, the highest standard deviation of mean was related to PDMS slab submerged in toluene $(1.286 \pm 0.0317)$ that was small enough $(<2.5 \%)$ to confirm the repeatability and reliability of the experiments.

As mentioned earlier, 1:20 PDMS slab had highest swelling ratio where submerged slabs in toluene and hexane with $128 \%$ and $105 \%$ increase in weight had the largest ratios, respectively. It means low cross linked elastomer (1:20 curing agent:base PDMS) swells more than other ratios where solvent molecules detach non-crosslinked oligomers from PDMS. Toluene and hexane swelled over $85 \%$ after $80 \mathrm{~min}$ while other solvents have less than $20 \%$ of swelling. In fact, PDMS with low polarity [43] contribution had higher swelling ratio in liquids with lower polarity index (i.e. toluene and hexane) while PDMS is more compatible with polar liquids (i.e. methanol, acetone, isopropanol). Solvents with high solubility in PDMS are not compatible with PDMS microfluidic platforms even for fast experiments. As can be seen, higher rate of swelling is related to the first $60 \mathrm{~min}$ and was decreased until equilibrium condition was achieved. For example, swelling was stopped after $350 \mathrm{~min}$ for 1:20 PDMS in toluene and 250 min for 1:20 ratio PDMS in hexane. Comparison between 1:10 and 1:2 PDMS slabs revealed that higher reagent to base ratio sample (1:2) with a rigid, fragile structure, had higher swelling ratio. Ratio of the curing agent to base affects the number of cross-links in cured PDMS and higher swelling ratio of PDMS in solvent, consequently [18]. It seems that 1:10 cross-linker to base reagent ratio was the preferential ratio for minimizing organic solvent absorption. Neither increasing nor decreasing the amount of cross-linker had any beneficial effects for solvent compatibility. This is in good agreement with previous studies that showed minimum swelling ratio for 1:10 PDMS sample [44].

Final swelling ratio as a function of solubility parameter is shown in Fig. 3. According to Fig. 2, the highest swelling ratios were related to 1:20 reagent to base ratio PDMS slab in toluene and hexane. These two had smaller interaction radius (Table 1) compared with other solvents. Moreover, effective factors on solubility parameter can affect the swelling ratio drastically. For instance, as represented in 
Fig. 2 Swelling ratio of PDMS chips in a Isopropanol, b Toluene, c Acetone, $\mathbf{d}$ Hexane, e Methanol. Error bars were calculated with repeatability of the experiment
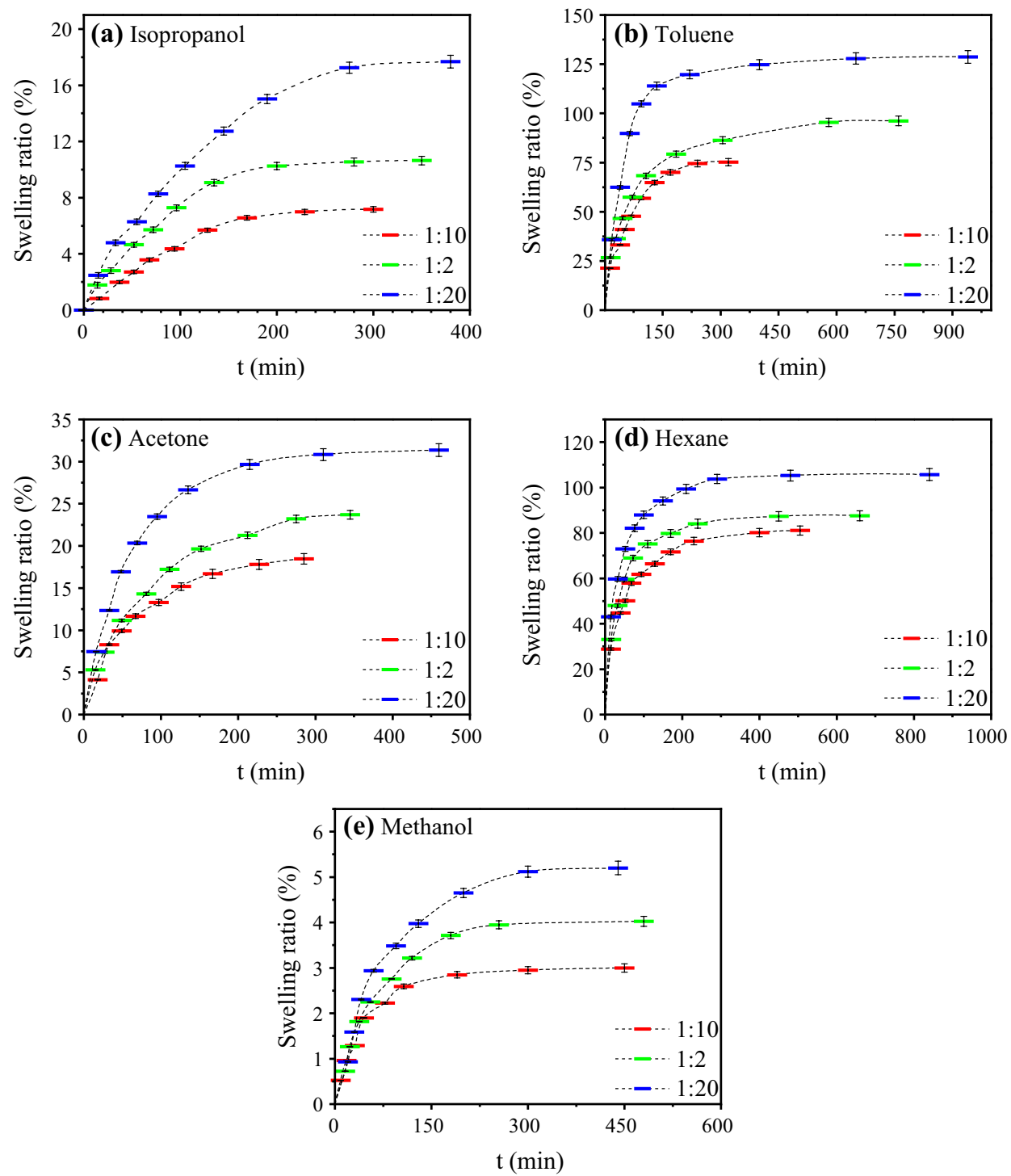

Table 1 [40], polar section of the acetone $\left(10.4 \mathrm{MPa}^{0.5}\right)$ is very different compared with hexane (i.e. $0.01 \mathrm{MPa}^{0.5}$ ) that caused large PDMS swelling difference of $105 \%$ and $31 \%$ between these solvents. In fact, low soluble liquids had high polar forces $\left(\delta_{p}\right)$ section $\left(\delta_{p}>10 \mathrm{MPa}^{0.5}\right)$ while highly soluble solvents had small polar forces $\left(<1.5 \mathrm{MPa}^{0.5}\right)$ that is closer to PDMS polar force. Figure 3 showed that different reagent to base PDMS samples for low soluble liquids such as methanol did not affect the swelling ratio.

Swelling ratio of FOTS coated PDMS slabs were measured for different curing agent:base ratios and shown in Fig. 4 for toluene as an example. PDMS coating followed the same steps for all the samples and each experiment was repeated three times to compare the mean and standard deviation values. Swelling ratios and standard error of means were in the same order of uncoated PDMS samples that means silane coating did not improve the solvent compatibility of the PDMS. It shows that coating with short-chain silane would not affect the swelling. In fact, the low wetting did not prevent the leakage of small solvent molecules into PDMS when immersed in the solvent. Kushmeric et al. [45] showed aggregates of deposited perfluorocyltrichlorosilane on the surface of PDMS, led to the degradation of expected hydrophobic surface property of treated PDMS.

\subsection{Diluted bitumen}

\subsubsection{Swelling ratio}

The main aim of this study is to determine if PDMS chips are suitable for evaluation of heavy oil and bitumen flow. Transparent nature of PDMS along with its flexibility makes it as a suitable option for micro structure 


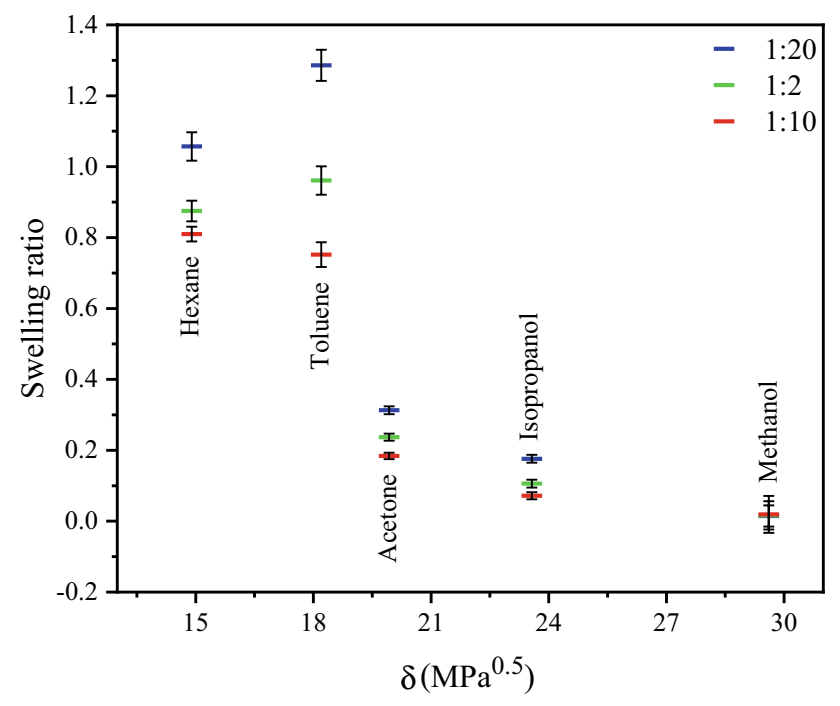

Fig. 3 Equilibrium swelling ratio of PDMS at different solvents (hallow shape: 1:2, filled shape: 1:10, and blank shape: 1:20 reagent to base ratio PDMS)

fabrication. However, the nature of bitumen with a wide range of components and presence of organic solvent such as hexane in some extraction methods such as Vapor Extraction (VAPEX) can affect the microchannel structure, size and even transparency. Therefore, swelling ratio of $1: 2$ and 1:10 curing agent to base PDMS samples were evaluated at diluted bitumen samples in hexane (10-50 wt\%). As shown in Fig. 5, higher swelling ratio for 1:2 PDMS slabs was notified compared with 1:10 samples. This means that 1:10 PDMS is still the optimum ratio. However, the swelling ratio of both PDMS samples (1:2 and 1:10) seems to be high and probably not good for heavy oil and bitumen related experiments. The higher swelling ratio was related to $10 \mathrm{wt} \%$ bitumen samples which means solvent had more effect on swelling compared with bitumen. However, higher equilibrium swelling ratio of $10 \mathrm{wt} \%$ bitumen sample compared with pure hexane swelling ratio revealed the effect of bitumen molecules sticking on the surface of PDMS slab. In fact, large bitumen molecules of bitumen along with solvent increased the swelling ratio but with increasing the concentration of bitumen, effect of solvent

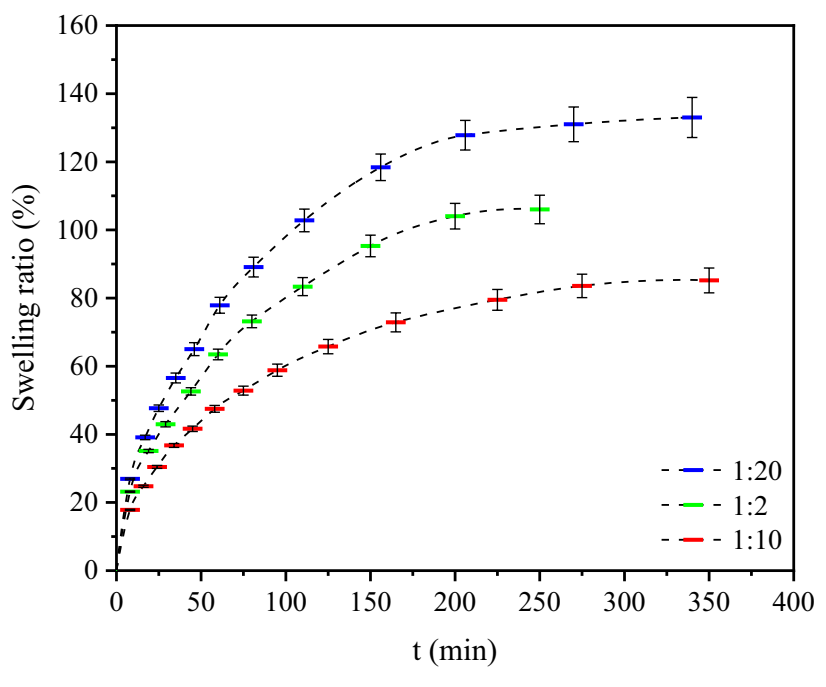

Fig. 4 Swelling ratio of FOTS coated PDMS slabs in toluene

molecules, as the dominant molecules, is reduced compared with pure solvent.

In order to have a better understanding about the deformation of the samples, PDMS microchannel was fabricated and diluted bitumen was injected into the microchannel. Prior to diluted bitumen injection, PDMS microchannel was examined with water with no destructive effect on PDMS using white light microscope coupled with CCD camera. According to time-lapsed images of capillary flow of water in PDMS microchannels (Fig. 6), deformation of channel and leakage was not monitored and water flowed through the microchannel. In fact, it confirmed PDMS microchannel is applicable in capillary-driven flow study of specific liquids. Moreover, capillary flow of diluted bitumen (10-60 wt\%) in glass etched microchannels was studied in our previous work and proved the application of glass microchannel for any kind of liquid due to glass stability and resistivity.

A quick and intense destabilization of glass-PDMS bonding led $50 \%$ diluted bitumen to draw out of the microchannel after injection. Capillary-driven flow of diluted bitumen in microchannel, leakage of the fluid, and glass-PDMS bonding area are represented in Fig. 7a. In fact, black area shows capillary-driven flow of diluted bitumen
Table 1 Solubility parameters and interaction radius of solvents and PDMS

\begin{tabular}{llllll}
\hline & $\delta_{d}\left(\mathrm{MPa}^{0.5}\right)$ & $\delta_{p}\left(\mathrm{MPa}^{0.5}\right)$ & $\delta_{h}\left(\mathrm{MPa}^{0.5}\right)$ & $\delta\left(\mathrm{MPa}^{0.5}\right)$ & $R_{i}\left(\mathrm{MPa}^{0.5}\right)$ \\
\hline Hexane [40] & 14.9 & 0.01 & 0.00 & 14.9 & 9.45 \\
Toluene [40] & 18.04 & 1.39 & 2.01 & 18.2 & 7.11 \\
Acetone [40] & 15.5 & 10.4 & 7 & 19.93 & 11.63 \\
Methanol [40] & 15.1 & 12.3 & 22.3 & 29.61 & 18.66 \\
Isopropanol [40] & 15.8 & 6.1 & 16.4 & 23.57 & 12.05 \\
PDMS [41] & 16.86 & 0.12 & 8.6 & 18.92 & - \\
\hline
\end{tabular}



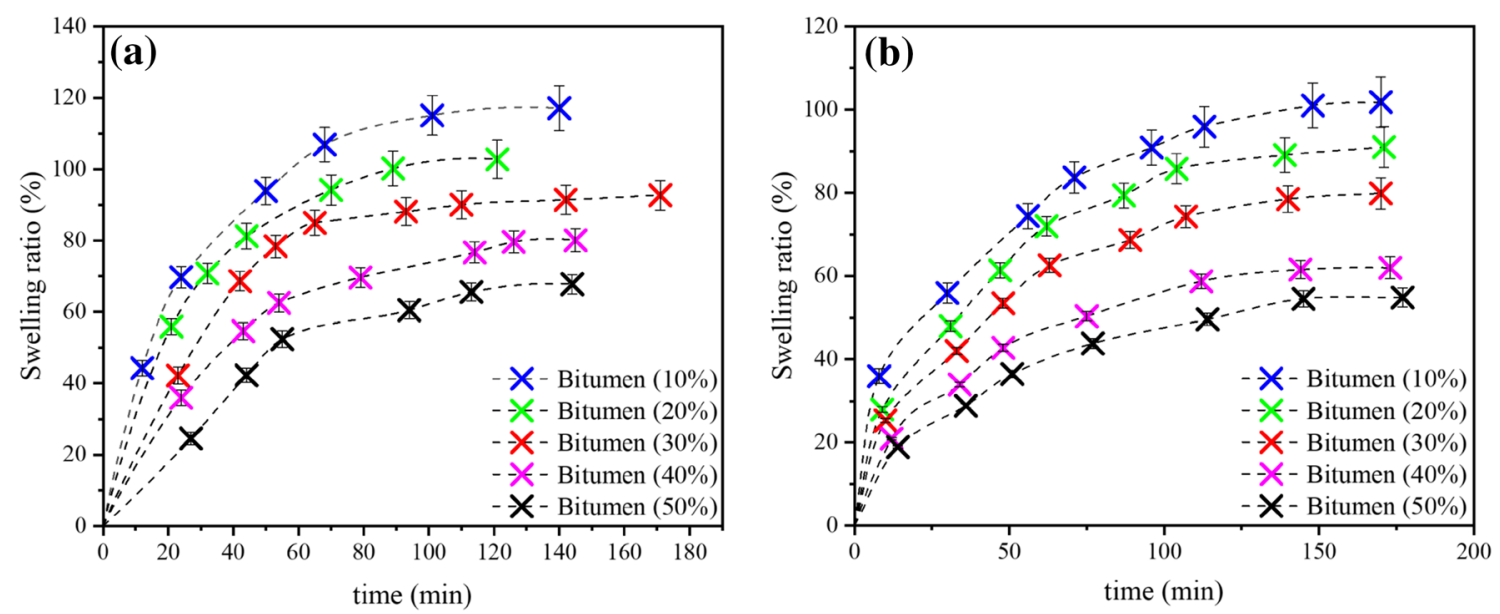

Fig. 5 Swelling ratio of diluted bitumen in PDMS, a 1:2, b 1:10

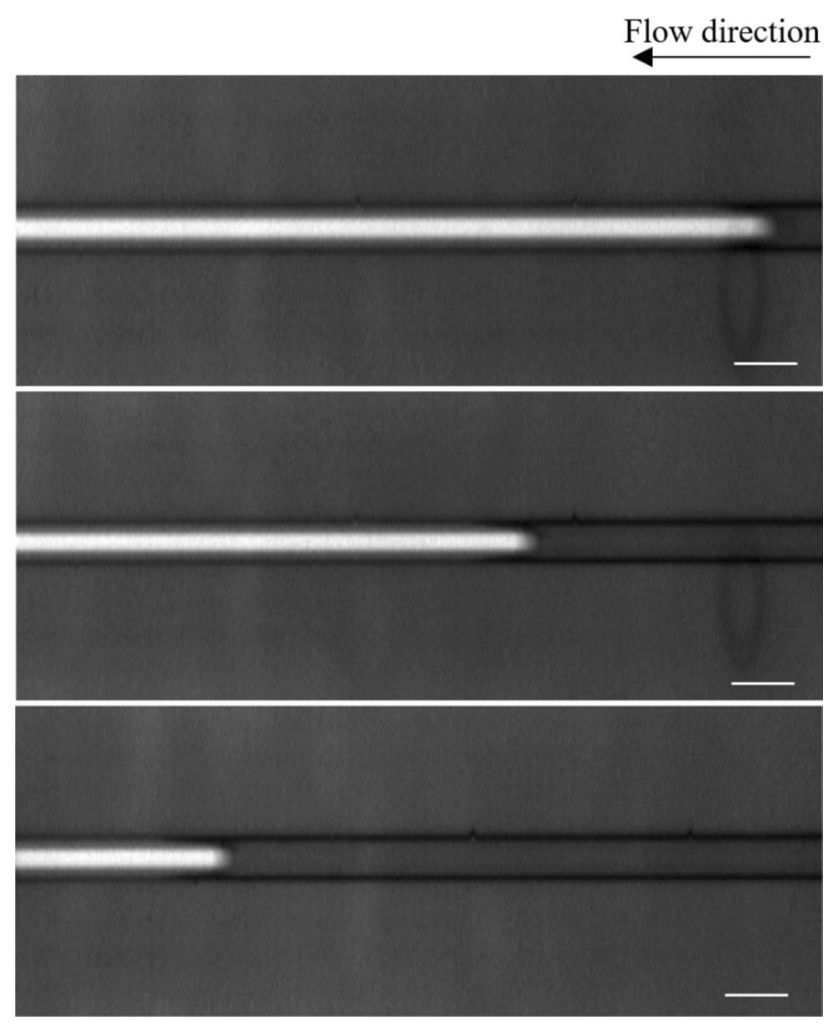

microchannel with PDMS deformation and leakage of bitumen in the glass-PDMS bonded area after $5 \mathrm{~min}$ is shown in Fig. 7b, c. Diluted bitumen leaked out of the injection reservoir and microchannel (Fig. 7b). As shown in Fig. 7c, PDMS microchannel was deformed and destabilized the glass-PDMS bonding and enclosed microchannel is not available anymore.

\subsubsection{Transparency and fluorescence intensity}

The UV-Vis transmittance spectra of 1:10 curing agent to base ratio PDMS is shown in Fig. 8 as a function of time at wavelength of $400 \mathrm{~nm}$ and based on three measurements at different positions of the sample. A higher transmittance reduction of PDMS was reported with increasing the concentration of bitumen. Transparency of $50 \%$ bitumen after $180 \mathrm{~min}$ was as low as $15 \%$ which is not enough for using PDMS microchannel in heavy oil and bitumen flow study.

As mentioned earlier, fluorescence microscopy is an efficient way to detect bitumen. Therefore, TIRF microscope was applied to detect bitumen on the surface layer of 1:10 PDMS in different bitumen samples (10-50 wt\%). PDMS slab was submerged into bitumen sample and bitumen content was evaluated at different times. Based on previous studies, it was understood that fluorescence from the hexane was not interfere with fluorescence from bitumen in our desired excitation and emission wavelength so all the fluorescence of the samples is related to bitumen. Fluorescence intensity of 1:10 PDMS in diluted bitumen (50 wt\%) with a $488 \mathrm{~nm}$ laser source and emission filter of 500-550 nm (green) after (a) 3, (b) 10 and (c) $15 \mathrm{~min}$ is shown in Fig. 9.

Fluorescent images showed that bitumen content of the PDMS surface is increasing fast that makes PDMS 


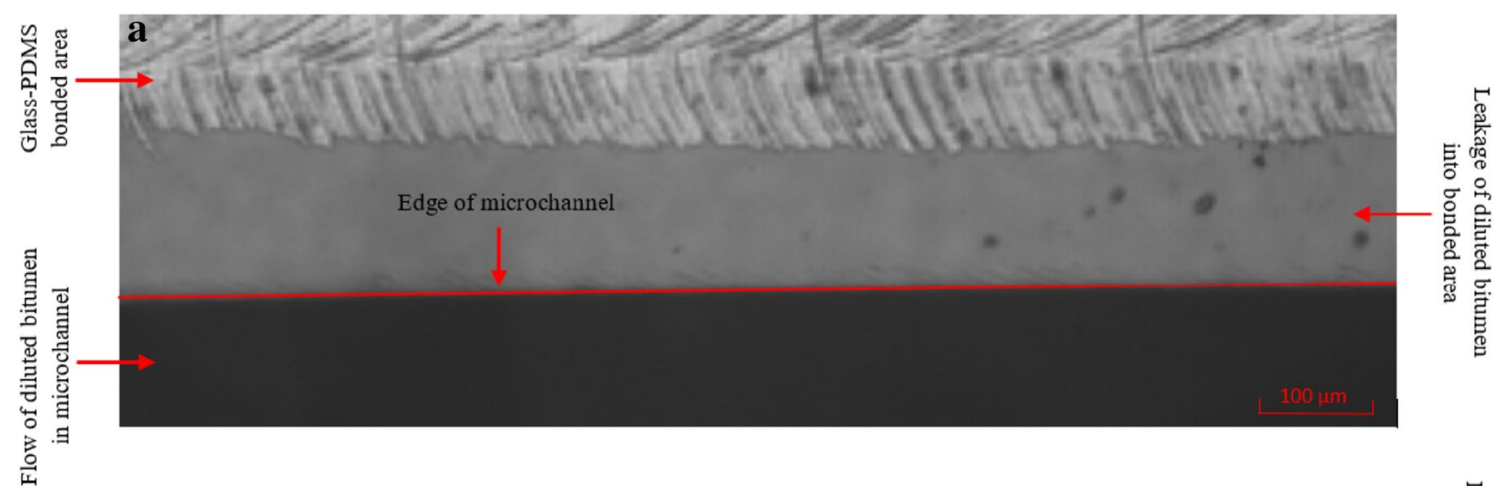

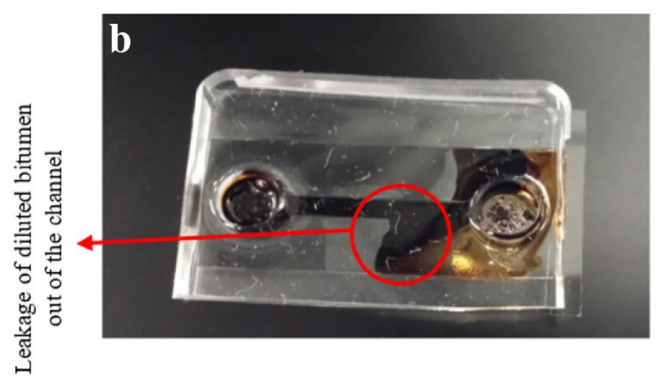

Fig. 7 Capillary flow of diluted bitumen in PDMS microchannel. a Microscopic view after $30 \mathrm{~s}$, black area shows bitumen flow in microchannel, dark grey area shows sample leakage, and light grey

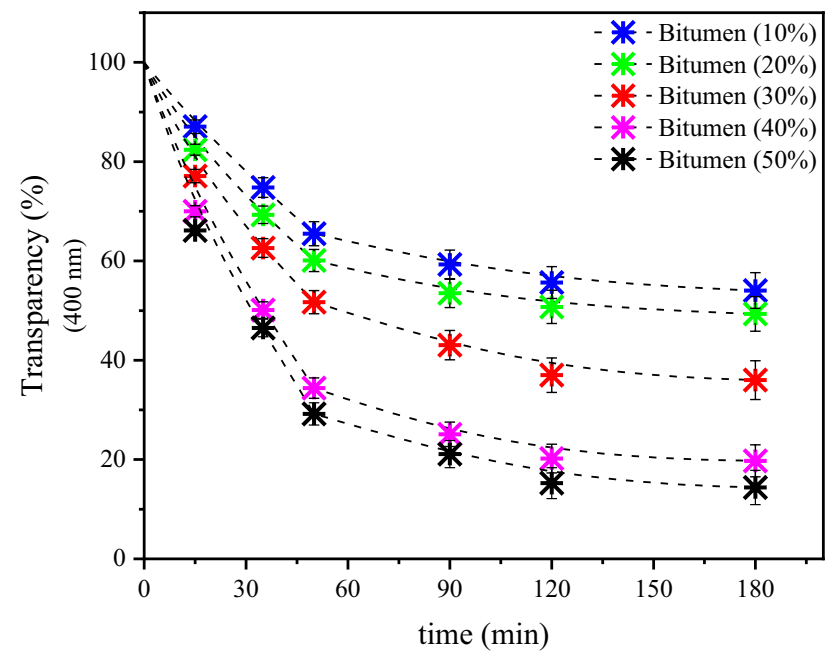

Fig. 8 UV-VIS spectrum of a $4 \mathrm{~mm}$ thick 1:10 PDMS slab at different bitumen concentration

inappropriate for micro scale monitoring of heavy oil and bitumen. Moreover, bitumen is increasing is size on the surface that could be related to bridging and aggregation of asphaltene and other components of the bitumen on the PDMS surface. However, larger size of bitumen molecules compared with pure solvents did not reduce the swelling rate of bitumen in PDMS slab area shows glass-PDMS bonding area, $\mathbf{b}$ top view of PDMS micromodel shows diluted bitumen leakage, and $\mathbf{c}$ side view after $5 \mathrm{~min}$

and PDMS microchannel. All in all, it seems dynamic of the swelling is fast and it is not possible to use PDMS either for pure solvent or high viscosity fluids such as bitumen and heavy oil.

\section{Conclusion}

Application of PDMS microchannel as a fast and cheap approach can improve our understanding of fluid flow in small scales such as porous media. Solubility parameters are good factors for swelling correlation of PDMS. It has been reported that PDMS has high swelling of pure solvents that makes it unsuitable for solvent related studies. This was confirmed with polar factor in solubility where similar polar factor leads to high swelling of hexane and toluene in PDMS. Swelling dynamic of pure liquids and large molecules such as bitumen along with its swelling ratio has not thoroughly evaluated yet. On the other hand, if dynamic of swelling is not very fast, PDMS microchannel can still be applicable for investigation of fluid flow. In this study, it was found that swelling of immersed PDMS into toluene and hexane was very fast with deformation of the PDMS slab (i.e. curved edges). Degree of swelling in other solvents was relatively small with faster steady state swelling. In addition, experimental solvent swelling of different PDMS (base to curing agent ratio) was measured 
Fig. 9 TIRF images of PDMS swollen at $50 \%$ bitumen solution a $t=3 \mathrm{~min}, \mathbf{b} t=13 \mathrm{~min}, \mathbf{c}$ $t=22 \min$
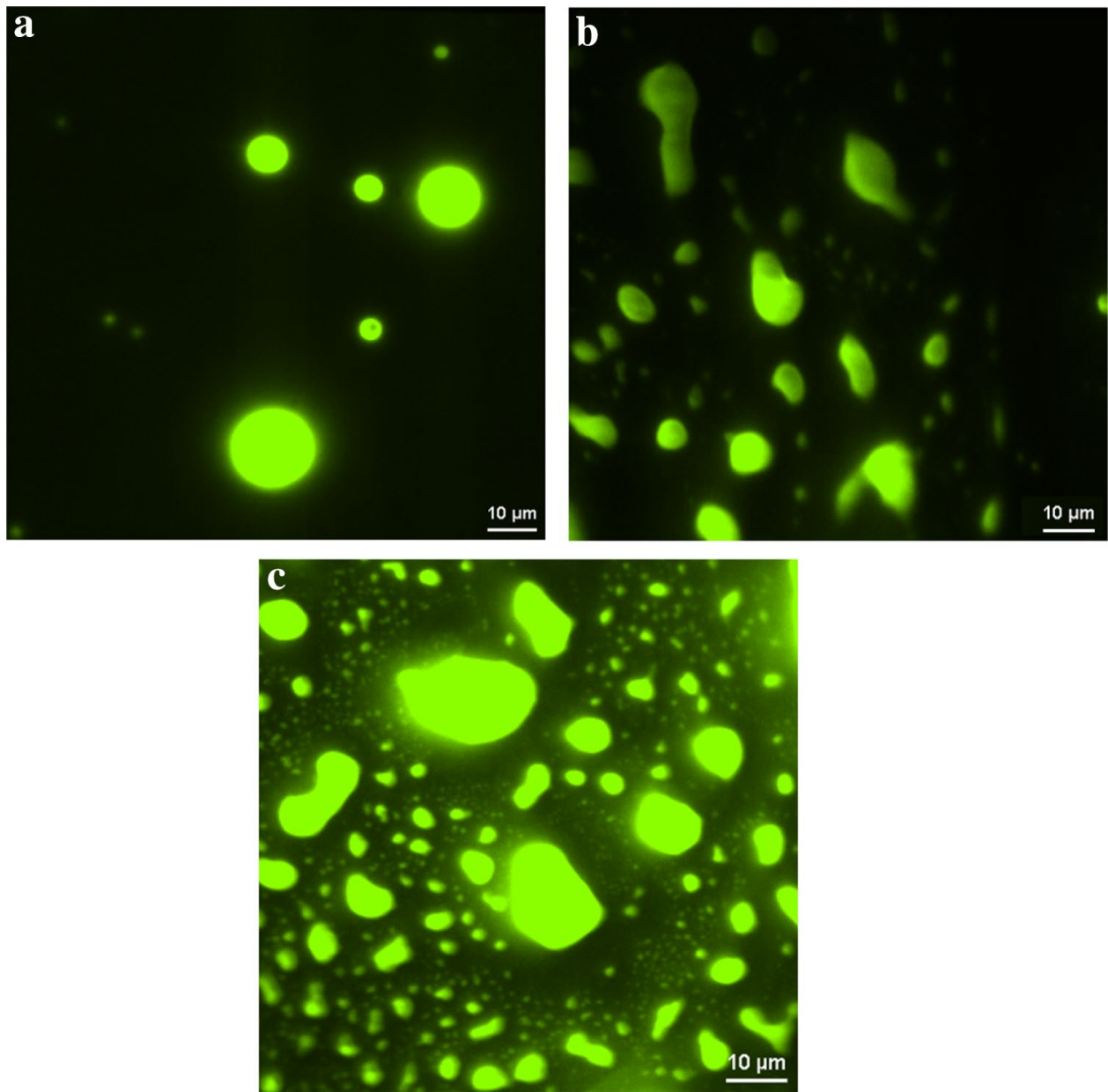

to find the best ratio for PDMS fabrication and 1:10 ratio PDMS had the minimum swelling ratio. The improvement of solvent compatibility of PDMS with silanization was evaluated using FOTS as a fast and chip method of surface modification. However, high level of swelling ratios was reported for coated PDMS slabs. This means that silanization is not a suitable approach for solvent compatibility enhancement.

Moreover, compatibility of PDMS with bitumen as a highly viscous and large molecule fluid was evaluated to see if PDMS is applicable for heavy oil and bitumen flow evaluation. Diluted bitumen (10-50 wt\%) was injected in PDMS fabricated microchannel and the shape of the microchannel was disturbed after injection of diluted bitumen quite fast and glass, PDMS bonding was broken. Deformation of microchannel along with fast dynamic of swelling mean that PDMS is not suitable for evaluation of flow and rheology of heavy oil and bitumen. Furthermore, transparency of 1:2 and 1:10 PDMS slabs were measured that higher concentration of bitumen led to lower transparency and poor bitumen flow visualization consequently. TIRF microscopy was the last step for evaluation of bitumen swelling dynamic. It clearly showed that bitumen concentration on the PDMS surface was increasing fast. High level of swelling, low transparency and high concentration of bitumen on the surface are solid reasons to believe that PDMS microchannels are limited to certain solvents and are not a potential replacement for glass microchannels for capillary flow evaluation of bitumen and heavy oil. Equilibrium swelling ratio of coted PDMS revealed that surface treatment with short chain silane did not improve the resistivity toward swelling.

Acknowledgements The authors would like to thank InnoTech Alberta and Natural Sciences and Engineering Research Council of Canada (NSERC) for funding. The authors also acknowledge Dr. HyunJoong Chung for his insightful comments and the use of silanization unit in his lab. The authors would like to thank Nicholas Moore for helping with experimental data.

Funding This study was funded by Alberta Innovates - Alberta Research Council Core Industry (AACl) PhD Program (Grant No. RES0024693). 


\section{Compliance with ethical standards}

Conflict of interest The authors declare that they have no conflict of interest.

\section{References}

1. Hu C, Morris JE, Hartman RL (2014) Microfluidic investigation of the deposition of asphaltenes in porous media. Lab Chip 14(12):2014-2022. https://doi.org/10.1039/C4LC00192C

2. Molla S, Mostowfi F (2017) Microfluidic PVT: saturation pressure and phase-volume measurement of black oils. SPE Reserv Eval Eng 20(1):233-239. https://doi.org/10.2118/170910-PA

3. Fisher R, Shah MK, Eskin D, Schmidt K, Singh A, Molla S, Mostowfi $F$ (2013) Equilibrium gas-oil ratio measurements using a microfluidic technique. Lab Chip 13:2623-2633. https://doi. org/10.1039/C3LC00013C

4. Keshmiri K, Pourmohammadbagher M, Huang H, Nazemifard N (2019) Microfluidics to determine the diffusive mass transfer of a low viscosity solvent into a high viscosity hydrocarbon. Fuel 235:1327-1336. https://doi.org/10.1016/j. fuel.2018.08.108

5. Lee H, Leeb SG, Doyle PS (2015) Photopatterned oil-reservoir micromodels with tailored wetting properties. Lab Chip 15(14):3047. https://doi.org/10.1039/c5lc00277j

6. Keshmiri K, Mozaffari S, Tchoukov P, Huang H, Nazemifard N (2016) Using microfluidic device to study rheological properties of heavy oil. Presented at American Institude of Chemical Engineering (AIChE) annual meeting, San Francico, USA, 14 Nov

7. Xu K, Liang T, Zhu P, Qi P, Lu J, Huh C, Balhoff M (2017) A 2.5-D glass micromodel for investigation of multi-phase flow in porous media. Lab Chip 17:640-646. https://doi.org/10.1039/ c6lc01476c

8. Keshmiri K, Huang H, Nazemifard N (2019) Microfluidic platform to evaluate asphaltene deposition during solventbased extraction of bitumen. Fuel 239:841-851. https://doi. org/10.1016/j.fuel.2018.11.044

9. Va $L$ (2016) Microfluidics: an enabling screening technology for enhanced oil recovery (EOR). Lab Chip 16(10):1777-1796. https://doi.org/10.1039/c6lc00318d

10. Lele P, Syed AH, Riordon J, Mosavat N, Guerrero A, Fadaei $H$, Sinton D (2018) Deformation of microdroplets in crude oil for rapid screening of enhanced oil recovery additives. J Pet Sci Eng 165:298-304. https://doi.org/10.1016/j.petrol.2018.02.009

11. Esquivel JP, Del Campo FJ, Gómez de la Fuente JL, Rojas S, Sabaté N (2014) Microfluidic fuel cells on paper: meeting the power needs of next generation lateral flow devices. Energy Environ Sci 7:1744-1749. https://doi.org/10.1039/C3EE44044C

12. Pasirayi G, Auger V, Scott $S$, Rahman P, Islam M, O'Hare L, Ali Z (2011) Microfluidic bioreactors for cell culturing: a review. Micro Nanosyst 3(2):137-160. https://doi.org/10.2174/18764 02911103020137

13. Reyes D, lossifidis D, Auroux PA, Manz A (2002) Micro total analysis systems. 1. Introduction, theory, and technology. Anal Chem 74(12):2623-2636. https://doi.org/10.1021/ac0202435

14. Coltro WKT, Lunte SM, Carrilho E (2008) Comparison of the analytical performance of electrophoresis microchannels fabricated in PDMS, glass, and polyester-toner. Electrophoresis 29(24):4928-4937. https://doi.org/10.1002/elps.200700897

15. Hashimoto M, Langer R, Kohane DS (2013) Benchtop fabrication of microfluidic systems based on curable polymers with improved solvent compatibility. Lab Chip 13(2):252-259. https ://doi.org/10.1039/c2lc40888k

16. Leclerc E, Sakai Y, Fujii T (2004) Microfluidic PDMS (Polydimethylsiloxane) bioreactor for large-scale culture of hepatocytes. Biotechnol Prog 20(3):750-755. https://doi.org/10.1021/ bp0300568

17. Yao M, Fang J (2012) Hydrophilic PEO-PDMS for microfluidic applications. J Micromech Microeng 22(2):025012

18. Lee JN, Park C, Whitesides GM (2003) Solvent compatibility of poly(dimethylsiloxane)-based microfluidic devices. Anal Chem 75(23):6544-6655. https://doi.org/10.1021/ac0346712

19. Ng J, Gitlin I, Stroock A, Whitesides G (2002) Components for integrated poly(dimethylsiloxane) microfluidic systems. Electrophoresis 23(20):3461-3473. https://doi.org/10.1002/15222683(200210)23:20\%3c3461:AID-ELPS3461\%3e3.0.CO;2-8

20. Johnston ID, McCluskey DK, Tan CKL, Tracey MC (2014) Mechanical characterization of bulk Sylgard 184 for microfluidics and microengineering. J Micromech Microeng 24:35017. https://doi.org/10.1088/0960-1317/24/3/035017

21. Xiang C, Yang N, Zhang R, Guo J, Huang H (2017) An ultraviolet-visible (UV) photometry system based on the PDMS-based microfluidic chip. Presented at 13 th global congress on manufacturing and management (GCMM 2016), Zhengzhou, China. https://doi.org/10.1051/matecconf/201710003036

22. Khanafer K, Duprey A, Schlicht M, Berguer R (2009) Effects of strain rate, mixing ratio, and stress-strain definition on the mechanical behavior of the polydimethylsiloxane (PDMS) material as related to its biological applications. Biomed Microdevice 11(2):503-508. https://doi.org/10.1007/s10544-008-9256-6

23. Wang Z (2011) Polydimethylsiloxane mechanical properties measured by macro-scopic compression and nanoindentation techniques. PhD diss., University of South Florida

24. Seghir R, Arscott S (2015) Extended PDMS stiffness range for flexible systems. Sens Actuators 230(1):33-39. https://doi. org/10.1016/j.sna.2015.04.011

25. Saryazdi F, Motahhari H, Schoeggl FF, Taylor SD, Yarranton HW (2013) Density of hydrocarbon mixtures and bitumen diluted with solvents and dissolved gases. Energy Fuels 27(7):36663678. https://doi.org/10.1021/ef400330j

26. Abate AR, Lee D, Do T, Holtzea C, Weitz DA (2008) Glass coating for PDMS microfluidic channels by sol-gel methods. Lab Chip 8:516-518. https://doi.org/10.1039/b800001h

27. Cho SH, Godin J, Lo Y (2009) Optofluidic waveguides in teflon AF-coated PDMS microfluidic channels. IEEE Photonics Technol Lett 21(15):1057-1059. https://doi.org/10.1109/LPT.2009.20222 76

28. Diaz-Quijada GA, Wayner DDM (2004) A simple approach to micropatterning and surface modification of poly(dimethylsiloxane). Langmuir 20(22):9607-9611. https:// doi.org/10.1021/la048761t

29. Khorasani M, Mirzadeh H (2004) Laser surface modification of silicone rubber to reduce platelet adhesion in vitro. J Biomater Sci Polym Ed 15(1):59-72. https://doi.org/10.1163/1568562043 22752237

30. Pan Z, Shahsavan H, Zhang W, Yang FK, Zhao B (2015) Superhydro-oleophobic bio-inspired polydimethylsiloxane micropillared surface via FDTS coating/blending approaches. Appl Surf Sci 324(1):612-620. https://doi.org/10.1016/j.apsus c.2014.10.146

31. Diebold RM, Clarke DR (2012) Smooth, aggregate-free selfassembled monolayer deposition of silane coupling agents on silicon dioxide. Langmuir 28(44):15513-15520. https://doi. org/10.1021/la303377w

32. Shende SS, Pendharker S, Jacob Z, Nazemifard N (2016) Total internal reflection fluorescence microscopy to investigate the distribution of residual bitumen in oil sands tailings. Energy 
Fuels 30(7):5537-5546. https://doi.org/10.1021/acs.energyfuel s.6b00745

33. Du Y, Xue Y, Frisch HL (1996) Physical properties of polymers handbook. AIP Press, Woodbury

34. Ougizawa T, Inoue T (1999) Miscibility and interfacial behavior in polymer-polymer mixtures, in polymer blends and alloys, chapter 3. Marcel Dekker Inc, New York

35. Rumens CV, Ziai MA, Belsey KE, Batchelor JC, Holder SJ (2015) Swelling of PDMS networks in solvent vapours; applications for passive RFID wireless sensors. J Mater Chem C 3:10091-10098. https://doi.org/10.1039/C5TC01927C

36. Hansen CM (2004) 50 Years with solubility parameters-past and future. Prog Organ Coat 51(1):77-84. https://doi.org/10.1016/j. porgcoat.2004.05.004

37. Su X, Shi B, Wang L (2015) Investigation on three-dimensional solubility parameters for explanation and prediction of swelling degree of polydimethylsiloxane pervaporation membranes. J Macromol Sci 54:1248-1258. https://doi.org/10.1080/00222 348.2015.1085272

38. Hansen CM (2007) Hansen solubility parameters: a user's handbook. CRC Press, Boca Raton

39. Kusano Y, Teodoru S, Hansen CM (2011) The physical and chemical properties of plasma treated ultra-high-molecular-weight polyethylene fibers. Surf Coat Technol 205:2793-2798. https:// doi.org/10.1016/j.surfcoat.2010.10.041

40. Bruke J (1984) Solubility parameters: theory and application. AIC book pap, group annual vol 3, pp 13-54. https://cool.conservati on-us.org/coolaic/sg/bpg/annual/v03/bp03-04.html. Accessed 20 May 2017

41. Soltane H, Roizard D, Favre E (2013) Effect of pressure on the swelling and fluxes of dense PDMS membranes in nanofiltration: an experimental study. J Membr Sci 435(15):110-119. https:// doi.org/10.1016/j.memsci.2013.01.053

42. Handle F, Füssl J, Neudl $S$, Grossegger D, Eberhardsteiner L, Hofko B, Hospodka M, Blab R, Grothe H (2016) The bitumen microstructure: a fluorescent approach. Mater Struct 49(12):167-180. https://doi.org/10.1617/s11527-014-0484-3

43. Diachun NA, Marcus AH, Fayer MD (1994) Dynamics in polydimethylsiloxane: the effect of solute polarity. J Am Chem Soc 116(3):1027-1032. https://doi.org/10.1021/ja00082a025

44. Campbell DJ, Rupe SB (2000) Solvent swelling and optical rotation demonstrated on the overhead projector. J Chem Educ 77(7):876. https://doi.org/10.1021/ed077p876

45. Kushmeric JG, Hankins MG, de Boer M, Clews PJ, Carpick R (2001) The influence of coating structure on micromachine stiction. Tribol Lett 10(1-2):103-108. https://doi.org/10.1023/A:10090 82530479

Publisher's Note Springer Nature remains neutral with regard to jurisdictional claims in published maps and institutional affiliations. 